\title{
A Comparison Between the Effectiveness of Full Outline of Unresponsiveness and Glasgow Coma Score at Neurosurgical Intensive Care Unit Patients
}

\author{
Nail Caglar TEMIZ1', Gulsah KOSE¹, Ozkan TEHLI' ${ }^{1}$, Cengizhan ACIKEL², Sevgi HATIPOGLU³ \\ ${ }^{1}$ University of Health Sciences, Gulhane Training and Research Hospital, Department of Neurosurgery, Ankara, Turkey \\ ${ }^{2}$ University of Health Sciences, Gulhane Training and Research Hospital, Department of Biostatistics, Ankara, Turkey \\ ${ }^{3}$ Near East University, Faculty of Health Sciences, School of Nursing, Lefkosa, Turkish Republic of North Cyprus
}

\section{ABSTRACT}

AIM: To evaluate the effectiveness and the use of Glasgow Coma Score (GCS) and Full Outline of Unresponsiveness (FOUR) score by nurses in the follow-up and evaluation of patients admitted to the neurosurgical intensive care unit for cranial surgery or head trauma.

MATERIAL and METHODS: The study was performed at a neurosurgical intensive care unit. Sample size was determined as 47 patients $(\alpha=0.05$, power $=0.95)$. The correlation coefficient less than 0.5 was accepted as weak. In the first 24 hours, Karnofsky Performance Scale was applied and the Acute Physiology and Chronic Health Evaluation II (APACHE II) Score calculated for patients who were admitted to the intensive care unit for cranial surgery or head trauma. Also FOUR and GCS were applied by two different nurses twice a day. Intraclass Correlation Coefficient, Pearson Correlation and Cronbach's Alpha Security Index analyses were used to evaluate the data.

RESULTS: Concordance was above 0.810 and correlation was above 0.837 between GCS and FOUR score evaluation results of nurses. Correlation of two different evaluation at every shift for GCS was 0.887 , and for FOUR was 0.827 and above. Karnofsky Performance Scale correlation with FOUR and GCS scores of patients at admission and discharge from the intensive care unit was 0.709 and above. The correlation between APACHE II and FOUR was 0.851; between APACHE II and GCS 0.853 . There was no difference between the evaluations of two scores and two nurses statistically.

CONCLUSION: Concordance between nurses was found high both for GCS and FOUR. The FOUR score is as effective as GCS on the follow-up of patients who are managed in the neurosurgical intensive care units.

KEYWORDS: Glasgow coma score, Full outline of unresponsiveness score, Neurosurgery, Intensive care unit

\section{INTRODUCTION}

$\mathrm{E}$ valuation of comatose patients in intensive care units is an important issue for neurosurgeons (3). However, there is no objective value such as body temperature or blood pressure to evaluate intensive care unit comatose patients, and therefore different scales are used (10). One of these scales is Glasgow Coma Score (GCS), which is known worldwide and commonly used to predict the level of, consciousness, mortality and morbidity $(4,9,10,13)$. Even though GCS is the first thing that comes to mind in the whole world, contradiction between observers, inability to evaluate verbal response on intubated, sedated and delirium patients, and absence of brainstem reflexes in the scale are the subjects which are criticized in the literature $(3,4,10,13)$. Also, changes in consciousness cannot be spotted early because of brainstem reflexes and respiration pattern shifts $(10,13)$. Therefore GCS cannot be the most trustworthy evaluation method $(10,13)$. 
Different scoring systems have therefore been developed to evaluate patients in time $(3,9)$. One of these scores is the Full Outline of Unresponsiveness (FOUR) Score that was developed by Wijdicks et al. in $2005(3,4,9,13,16)$. There are 4 headers (eye response, motor response, brainstem reflexes, respiration) and scores from 0 to 4 , to evaluate patients with FOUR which was recommended by the European Society of Intensive Care Medicine (ESICM)(3,4,9,13). Therefore using the FOUR score ensures that brainstem reflexes, respiration pattern and verbal response that are supplementary data for patient evaluation, absent in GCS, can be used $(3,4,9)$. The FOUR score has advantages and still investigated by different countries and clinics (10). In some studies, there was no sample calculation for comparing GCS and FOUR, no surveys for GCS and FOUR at the same time, studies reported just for the first 24 hours and only traumatic brain injury patients or intubated patients had been included $(1,4,9,10,14)$. There is no survey that includes all cases such as brain tumor patients or head trauma patients and lasts longer than one day. Therefore, our aim was to assess the effectiveness of using GCS and FOUR by nurses on the follow-up and evaluation of patients who are admitted to the intensive care unit because of brain tumor or head trauma, in this study.

\section{MATERIAL and METHODS}

\section{Study Design and Samples}

This single center, prospective, cross-sectional study was performed between July 2012 and July 2013. Sample size was determined as 47 patients $(\alpha=0.05$, power $=0.95$, kappa $=$ $0.9)$. The correlation coefficient less than 0.5 was accepted as weak.

Inclusion criteria: Being older than 18 years, being admitted to intensive care unit because of brain tumor or head trauma.

Exclusion criteria: Being younger than 18 years, death within the first 24 hours.

\section{Data Collection}

The total form of demographic data that was developed by researchers, Karnofsky Performance Scale, Acute Physiology and Chronic Health Evaluation II (APACHE II) score, GCS and FOUR score were used in order to collect data. We evaluated patients admitted to the neurosurgical intensive care unit due to brain tumor or head trauma, and used the Karnofsky Performance Scale for functionality, APACHE II score for mortality, and FOUR and GCS for consciousness and coma level. We used GCS and FOUR on patients until discharge from the intensive care unit, twice a day, in the morning and evening, every day. FOUR and GCS assessments have been independently performed by responsible researchers and intensive care unit nurses.

\section{Ethical Considerations}

Initially, the ethical approval was obtained from the local ethical committee of our institution and written permission for this study was obtained from the department of neurosurgery (246-12/1539-546). All patient relatives and nurses were informed about this study, and written permission was obtained for inclusion in this study.

\section{Data Analysis}

The SPSS for Windows Ver. 15.00 (SPSS Inc., Chicago, IL, USA) program was used for the statistical analysis of the data. Compliance of measured or calculated values with a normal distribution was evaluated with the Shapiro-Wilks test. Intraclass Correlation Coefficient, Pearson correlation and Cronbach's alpha analysis were used for the analysis of the data. A $p$ value $\leq 0.05$ was accepted as an indicator of significance in all comparisons.

\section{RESULTS}

Twenty (42.5\%) of the 47 patients were female, 27 (57.5\%) patients were male. The mean age of patients was $46.68 \pm 20.40$ years in this study. Minimum length of stay in the intensive care unit was 1 day and the maximum was 7 days. Most of the patients $(60.5 \%)$ stayed in the intensive care unit because of an intracranial mass lesion. In the follow-up period, 6 patients received mechanical ventilation support, 34 patients were operated and 2 patients had complications. Mean Karnofsky score of the patients was $63.16 \pm 27.42$ at admission and $88.86 \pm 19.82$ at discharge. Minimum APACHE II score was 2, maximum score was 38 and minimum estimated death rate was $3.30 \%$, maximum was $88.40 \%$ for APACHE.

Coefficient of concordance was above 0.810 and correlation was above 0.837 between GCS and FOUR score evaluation results of nurses in every shift. Correlation of two different evaluation at every shift for GCS was 0.887 , and for FOUR it was 0.827 and above. Cronbach alpha coefficient was 0.940 for GCS, and 0.905 and above for FOUR.

Correlation between Karnofsky score and FOUR mean score of patients at admission to intensive care unit was 0.718 , and correlation between mean GCS was 0.771 . Correlation between Karnofsky score and FOUR mean score of patients at discharge from intensive care unit was 0.734 , and correlation between mean GCS was 0.709. Correlation between APACHE II score and FOUR-GCS scores relationship was 0.851 for FOUR and 0.853 for GCS.

\section{DISCUSSION}

Consciousness evaluation is the basic and important part of the neurological examination $(1,5,15)$. Grading the level of consciousness makes communication between medical staff more powerful and effective $(10,15)$. GCS and FOUR score are the most common used scales for this purpose $(1,5,14)$.

Verbal response of GCS cannot be evaluated on intubated, sedated and mechanical ventilation-supported patients $(1,8,14)$. Also other parameters like brainstem reflexes and respiration pattern cannot be evaluated by GCS (8). These unavailabilities may cause wrong decisions about evaluating the consciousness state, comatose level and mortality $(1,14)$. Neurological progress and evaluation can be done easier with the FOUR score that has been developed because of the disadvantages of GCS and more accurate decisions can be made $(1,8,14,15)$. In this study, where GCS and FOUR scores have been compared in brain tumor or head trauma patients at the intensive care unit, we found that the FOUR score is as 
Temiz NC. et al: Comparison Between FOUR and GCS

effective and trustworthy as GCS. Other reports also reveal the same thing as our study $(1,4,7-9,13)$.

FOUR and GCS scores have been found highly interclass correlation coefficient between practitioners. These were done by nurses twice a day. Also correlation for both scales was quite strong. Other studies have stated that coefficient of concordance and correlation between practitioners were high as in our work $(5,7,9,10,12-14)$. This conclusion is thought to be helpful for decreasing mistakes when evaluating intensive care unit patients and a more accurate evaluation can be made.

Scoring systems are frequently used to predict mortality by evaluating the severity of disease in intensive care units $(1,11)$. GCS is one of the most commonly used scales for this reason $(1,14)$. Other than GCS, Karnofsky Performance Scale (KPS) and APACHE II are used strengthen the prediction for intensive care unit patients $(11,14)$. Therefore, patient mortality rates and functionality were evaluated in our study, to evaluate accuracy of GCS and FOUR regarding mortality prediction. Evaluation results showed that concordance between FOUR, GCS and APACHE II, KPS was high. This finding shows that FOUR is as effective as GCS to predict mortality rate in intensive care unit patients. Other authors revealed that the concordance between FOUR and GCS is high to predict mortality rates, as in our study $(1,2,4,5,10,12)$.

There are some limitations of our study. One of the most important limitations is that it was done at one center and a single intensive care unit. We used one center and one intensive care unit because the FOUR score is not as widely used as GCS and patient follow-up must be done regularly. It is thought that including several centers and different intensive care units will provide more accurate findings. Another limitation of the study is that only nurses were evaluated. If physicians who work in different clinics and different intensive care units are included at other studies, more definitive conclusions can be reached and awareness of FOUR can be increased.

\section{CONCLUSION}

Nowadays, there is an argument suggesting that GCS is not trustworthy to assess consciousness of the intensive care unit patients, especially mechanical ventilation-supported patients. The inability to assess the patient's verbal response, brainstem reflexes and respiration pattern causes the GCS score to decrease. This situation results in misinterpretation of the patient. Despite all disadvantages, GCS is affirmed as the gold standard for evaluating the consciousness and comatose level of patients. The FOUR score has been developed alternatively and our findings suggest that it is highly reliable and easy to practice. Especially if there is no verbal response as in intubated, sedated or delirium patients, FOUR provides reliable information about the neurological conditions of these patients. Our study showed that the FOUR score can be safely determined by different practitioners. For this reason, we suggest that, the FOUR score is safer and more appropriate in patients with verbal non-response and altered respiration pattern.

\section{REFERENCES}

1. Baratloo A, Shokravi M, Safari S, Aziz AK: Predictive value of Glasgow Coma Score and Full Outline of Unresponsiveness Score on the outcome of multiple trauma patients. Arch Iran Med 19(3): 215-220, 2016

2. Chen B, Grothe $\mathrm{C}$, Schaller K: Validation of new neurological score (FOUR score) in the assessment of neurosurgical patients with severely impaired consciousness. Acta Neurochir 155:2133-2139, 2013

3. Fischer $M$, Rüegg $S$, Czaplinski $A$, Strohmeier $M$, Lehmann A, Tschan F, Hunziker PR, Marsch SC: Inter-rater reliability of the Full Outline of UnResponsiveness score and the Glasgow Coma Scale in critically ill patients: A prospective observational study. Crit Care 14(2): R64, 2010

4. Gorji MA, Gorji AM, Hosseini SH: Which score should be used in intubated patients' Glasgow coma scale or full outline of unresponsiveness? Int J Appl Basic Med Res 5(2):92-95, 2015

5. Gujjar AR, Jacob PC, Nandhagopal R, Ganguly SS, Obaidy A, Al-Asmi AR: Full outline of unresponsiveness score and Glasgow coma scale in medical patients with altered sensorium: Interrater reliability and relation to outcome. J Crit Care 28(3): 316.e1-8, 2013

6. Hickisch A, Holmefur M: Swedish translation and reliability of the full outline of Unresponsiveness Score. J Neurosci Nurs 48(4): 195-205, 2016

7. Jalali R, Rezaei M: A Comparison of the Glasgow Coma Scale Score with full outline of Unresponsiveness Scale to predict patients' traumatic brain injury outcomes in intensive care units. Crit Care Res Pract 2014:289803, 2014

8. Johnson VD, Whitcomb J: Neuro/trauma intensive care unit nurses' perception of the use of the full outline of Unresponsiveness Score versus the Glasgow Coma Scale when assessing the neurological status of intensive care unit patients. Dimens Crit Care Nurs 32(4):180-183, 2013

9. Khanal K, Bhandari SS, Shrestha N, Acharya SP, Marhatta MN: Comparison of outcome predictions by the Glasgow coma scale and the full outline of UnResponsiveness score in the neurological and neurosurgical patients in the Intensive Care Unit. Indian J Crit Care Med 20(8):473-476, 2016

10. Kramer AA, Wijdicks EF, Snavely VL, Dunivan JR, Naranjio LL, Bibel S, Rohs T, Dickess SM: A multicenter prospective study of inter-observer agreement using the full outline of Unresponsiveness score coma scale in the intensive care unit. Crit Care Med 40(9):2671-2676, 2012

11. Le Gall JR: The use of severity scores in the intensive care unit. Intensive Care Med 31:1618-1623, 2005

12. McNett MM, Amato S, Philippbar SA: A comparative study of Glasgow Coma Scale and full outline of Unresponsiveness Scores for predicting long-term outcome after brain injury. J Neurosci Nurs 48(4): 207-214, 2016

13. Peng J, Deng Y, Chen F, Zhang X, Wang X, Zhou Y, Zhou H, Qu B: Validation of the Chinese version of the FOUR score in the assessment of neurosurgical patients with different level of consciousness. BMC Neurology 15:254, 2015

14. Saika A, Bansal S, Philip M, Devi BI, Shukla DP: Prognostic value of FOUR and GCS scores in determining mortality in patients with traumatic brain injury. Acta Neurochir 157:13231328,2015

15. Sepahvand E, Jalali R, Mirzaei M, Ebrahimzadeh F, Ahmadi M, Amraii E: Glasgow Coma Scale versus full outline of UnResponsiveness Scale for prediction of outcomes in patients with traumatic brain injury in the intensive care unit. Turk Neurosurg 26(5): 720-724, 2016

16. Wijdicks EF, Bamlet WR, Maramattom BV, Manno EM, McClelland RL: Validation of a new coma scale: The FOUR score. Ann Neurol 58:585-593, 2005 